\title{
Quality of cardiopulmonary resuscitation in out-of-hospital cardiac arrest before and after introduction of a mechanical chest compression device, LUCAS-2; a prospective, observational study
}

Tinne Tranberg ${ }^{1 *}$, Jens F Lassen ${ }^{1 \dagger}$, Anne K Kaltoft ${ }^{1+}$, Troels M Hansen ${ }^{2,3,4+}$, Carsten Stengaard ${ }^{1+}$, Lars Knudsen ${ }^{4 \dagger}$, Sven Trautner ${ }^{5+}$ and Christian J Terkelsen ${ }^{1+}$

\begin{abstract}
Background: Mechanical chest compressions have been proposed to provide high-quality cardiopulmonary resuscitation (CPR), but despite the growing use of mechanical chest compression devices, only few studies have addressed their impact on CPR quality. This study aims to evaluate mechanical chest compressions provided by LUCAS-2 (Lund University Cardiac Assist System) compared with manual chest compression in a cohort of out-of-hospital cardiac arrest (OHCA) cases.

Methods: In this prospective study conducted in the Central Denmark Region, Denmark, the emergency medical service attempted resuscitation and reported data on 696 non-traumatic OHCA patients between April 2011 and February 2013. Of these, 155 were treated with LUCAS CPR after an episode with manual CPR. The CPR quality was evaluated using transthoracic impedance measurements collected from the LIFEPAK 12 defibrillator, and the effect was assessed in terms of chest compression rate, no-flow time and no-flow fraction; the fraction of time during resuscitation in which the patient is without spontaneous circulation receiving no chest compression.

Results: The median total episode duration was 21 minutes, and the episode with LUCAS CPR was significantly longer than the manual CPR episode, 13 minutes vs. 5 minutes, $\mathrm{p}<0.001$. The no-flow fraction was significantly lower during LUCAS CPR (16\%) than during manual CPR (35\%); difference 19\% (95\% Cl: $16 \%$ to 21\%; $p<0.001$ ). No differences were found in pre- and post-shock no-flow time throughout manual CPR and LUCAS CPR. Contrary to the manual CPR, the average compression rate during LUCAS CPR was in conformity with the current Guidelines for Resuscitation, 102/minute vs. 124/minute, $p<0.001$.

Conclusion: Mechanical chest compressions provided by the LUCAS device improve CPR quality by significantly reducing the NFF and by improving the quality of chest compression compared with manual CPR during OHCA resuscitation. However, data on end-tidal $\mathrm{CO}_{2}$ and chest compression depth surrogate parameters of CPR quality could not be reported.
\end{abstract}

Keywords: Out-of-hospital cardiac arrest (OHCA), Resuscitation, CPR quality, Mechanical chest compression

\footnotetext{
* Correspondence: tinne.tranberg@clin.au.dk

${ }^{\dagger}$ Equal contributors

'Department of Cardiology, Aarhus University Hospital, Aarhus, Denmark

Full list of author information is available at the end of the article
} 


\section{Introduction}

High-quality chest compression and early defibrillation are particularly essential for survival outcome after outof-hospital cardiac arrest (OHCA) [1]. Recent guidelines for resuscitation underline the importance of quality chest compressions and of minimising the time with no chest compressions during cardiopulmonary resuscitation (CPR) [2]. However, studies have shown that chest compressions performed by health care professionals do not meet the recommendations for compression rate, depth and continuity, which results in considerably longer no-flow times than necessary and desirable [3,4].

To address these shortcomings, a mechanical chest compression device, LUCAS-2 (Lund University Cardiopulmonary Assist System), has been developed (Figure 1A). The LUCAS device has been proposed to provide high-quality chest compressions whereby the interruptions seen with manual CPR [5] may be avoided; and randomised animal studies have demonstrated significantly better coronary perfusion pressure and cerebral artery blood flow when LUCAS-2 is used than when manual CPR is performed [6].

Despite the growing use of these devices, randomised studies in humans have not been able to show better outcome for OHCA patients resuscitated with mechanical chest compressions [7-9]. Research comparing the effect of manual CPR and mechanical CPR in the same patient is limited. The present study aimed to implement LUCAS in the physician-manned prehospital critical care teams and the helicopter emergency medical service (HEMS) and to evaluate if the mechanical chest compressions provided by LUCAS improve CPR quality compared with manual chest compressions in OHCA patients.

\section{Patients and methods}

\section{Setting}

The emergency medical service (EMS) in the Central Denmark Region is organised as a two-tier system; it operates a double-dispatch service that covers an area of $13,142 \mathrm{~km}^{2}$ inhabited by a total of 1.3 million people. The first tier consists of 75 conventional ambulances manned with two EMS providers. The second tier consists of nine physician-manned prehospital critical care teams and one HEMS available 24/7. The prehospital critical care teams and the HEMS have the competency to provide advanced life support (ALS). ALS includes the potential use of the LUCAS device. Conventional ambulances provide basic life support (BLS) and defibrillation only.

The EMS in the Central Region Denmark has a standardised pre-hospital-resuscitation protocol, which was

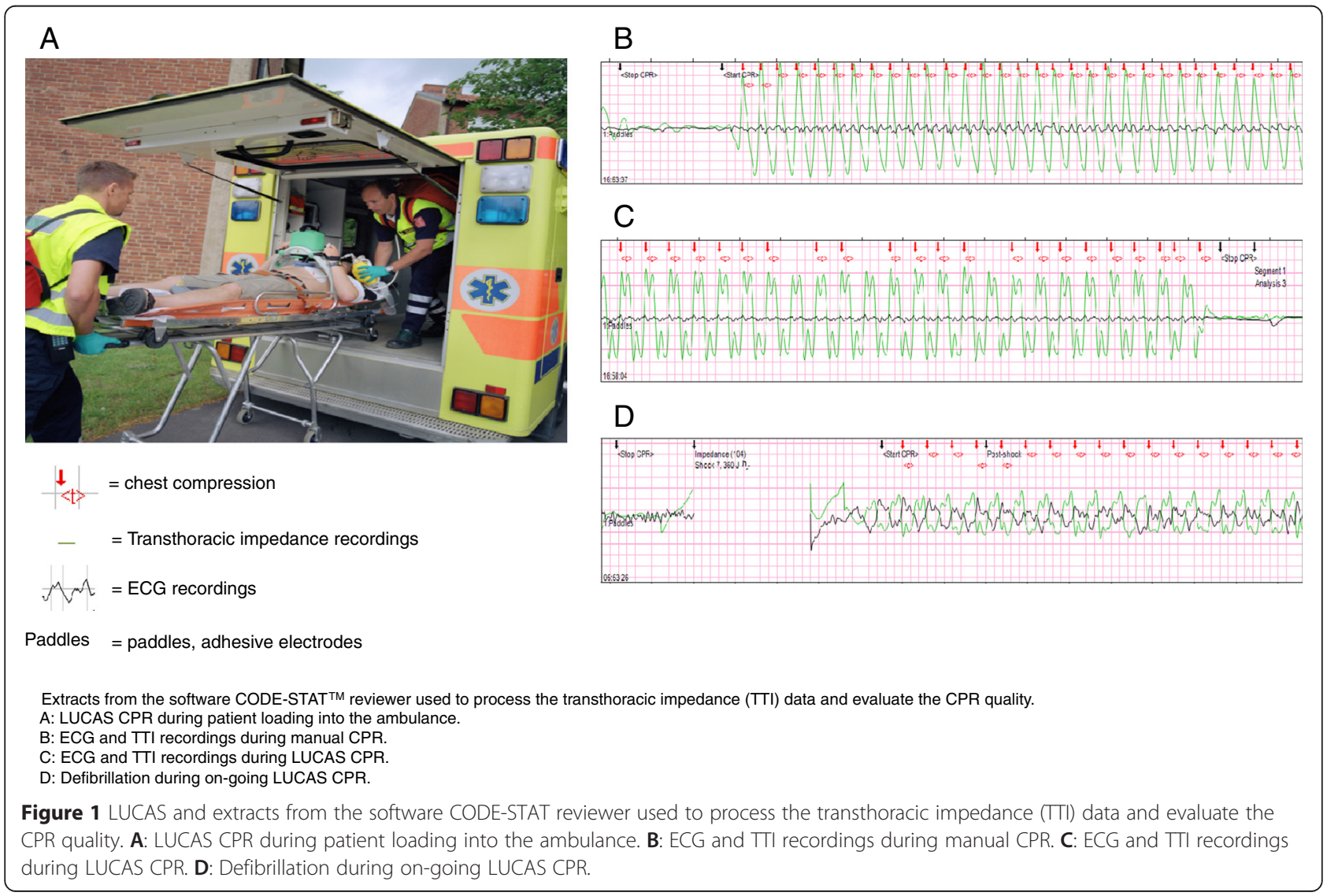


strictly adhered to in this study. A conventional ambulance is dispatched to all emergencies including OHCA. It normally arrives at the scene as a first responder. The prehospital critical care team or the HEMS is dispatched to patients with presumed OHCA as determined by the dispatcher triage and according to availability. All cardiac arrest patients are treated according to the 2010 Guidelines for Resuscitation. During the entire study period, the physicians of the prehospital critical care team and the HEMS serve jointly as the decision-maker who decides whether or not the LUCAS device is to be used in the attempt of resuscitation. In patients receiving LUCAS CPR, the EMS providers initiate the BLS until the arrival of the physician-manned mobile prehospital critical care team bringing the LUCAS device. Manual CPR is continued when the LUCAS device is being deployed; and in order to minimise the interruptions in $\mathrm{CPR}$, it is only paused briefly when the back plate is inserted and the arms positioned. When the prehospital critical care team or the HEMS are involved, the patient is transported directly to the tertiary university hospital's Heart Centre, which is staffed with dedicated anaesthesiologists, cardiologists and surgeons. Provided that active treatment is indicated, acute coronary angiography (CAG) and/or percutaneous coronary intervention (PCI) is performed, and therapeutic hypothermia is applied according to international guidelines. The patients are subsequently treated in the Cardiac Intensive Care Unit which is staffed by anaesthesiologists and cardiologists. However, if the prehospital critical care team or the HEMS are unable to assist the EMS providers, the operating procedure for the EMS providers state that after three defibrillation attempts or in the case of a nonshockable rhythm after three 2-minute cycles of CPR and persisting cardiac arrest, the patient is transported to the nearest hospital.
A total of nine LUCAS devices were introduced in the prehospital critical care team and the HEMS. Prior to this introduction, the physicians and the EMS providers were informed about the study protocol and the procedures, and they were instructed how to handle the LUCAS device. Furthermore, they accomplished a manikin-scenariotraining-session, which enabled them to use the device correctly, i.e. to deploy the device within 20 seconds while minimising interruptions in CPR. The physicians and the EMS providers who did not have the opportunity to attend the training session were trained and accredited by one of the physicians who took part in the session.

\section{Study design}

The inclusion criteria for the present study were nontraumatic OHCA, age above 18 years and OHCA occurring between 1 October 2011 and 31 January 2013. Exclusion criteria were pregnancy, trauma, intoxication, inability to attach the LUCAS device to the patient, patients with missing data and total resuscitation episode $<2$ minutes.

Data were collected prospectively and registered according to the Utstein templates for resuscitation registries [10] (Figure 2). The Danish Data Protection Agency (file number: 2013-41-1758) and The National Board of Health approved the present study, and the Regional Ethics Committee concluded that no formal approval was necessary because the study was designed as a quality-control study.

\section{Data collection}

Standard LIFEPAK 12 defibrillators (Physio-Control, Redmond, WA, USA) were used. De defibrillators continuously measure the transthoracic impedance (TTI) by applying a near constant current across the defibrillations pads. After a CPR effort, the ECGs and the TTI data of

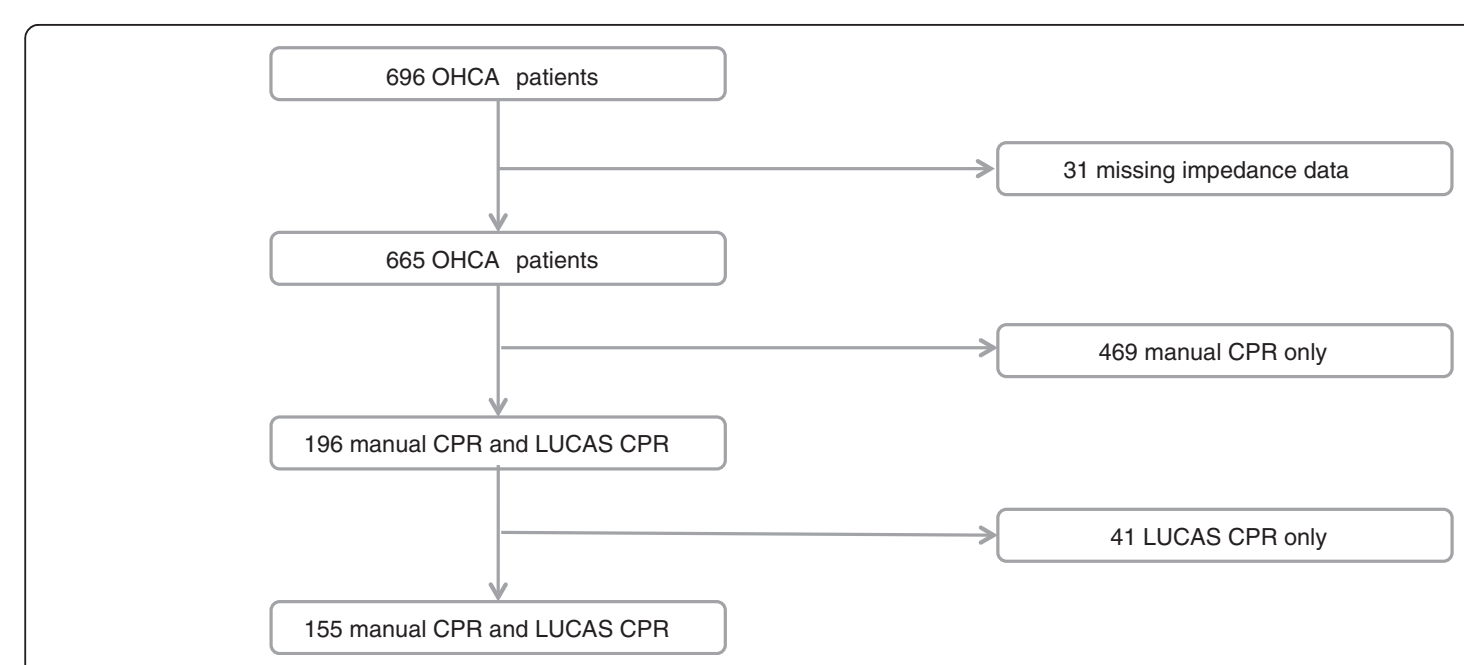

Figure 2 Study population. 
the OHCA case were transferred to a central server at the tertiary hospital; Aarhus University Hospital, Denmark. Furthermore, the prehospital critical care teams and the HEMS filled out a separate study form regarding the endtidal $\mathrm{CO}_{2}\left(\mathrm{ETCO}_{2}\right)$, which served as a surrogate marker of CPR quality.

The software programme CODE-STAT ${ }^{\text {Tn }}-8$ (PhysioControl Inc., Redmond, WA) was used to process the TTI data (Figure 1B, C, D). The software automatically annotates the chest compressions; however, each OHCA case was verified at the level of single compressions. Any incorrect automatic annotations were deleted, new annotations were added when annotations were missing; and the system calculated pre-shock and post-shock pauses, the compression rate and the actual number of compressions per minute, no-flow time (NFT) and no-flow fraction (NFF). The NFT, defined as the time without return of spontaneous circulation (ROSC) and the time without chest compressions, is a validated measure of the CPR quality, and it is reported according to previously published definitions [11-13]. The term NFF is defined as NFF $=$ (NFT/episode duration - time with ROSC). The NFF represents the proportion of interruptions in CPR during the episode. The actual number of chest compressions delivered per minute represents both the compression rate and the pauses in the compressions.

We defined the CPR-pause interval as the time from the trailing edge of the last chest compression to the leading edge of the next chest compression. Mechanical compressions were distinguished from the manual compressions by their highly regular morphology (Figure 1C). Usable data files included TTI data for the entire episode, from the first manual chest compression to the end of the final mechanical chest compression. The case was excluded if the total episode was shorter than 2 minutes.

Additionally, nationally adapted Utstein style forms filled out by the EMS personnel were used to obtain data on bystander CPR, OHCA location and the use of an automated external defibrillator (AED) prior to arrival of the EMS and whether OHCA was witnessed or unwitnessed. Data on the patients who achieved ROSC, survived to hospital discharge and survived after 30 days were collected from the OHCA registration form, ambulance records and hospital records.

\section{The properties of LUCAS-2}

LUCAS is a chest compression system that provides both active compression and decompression of the chest wall back to neutral position (Figure 1A). The first generation of the device, LUCAS-1, was driven by compressed air which was superseded by a battery-driven device in 2009. The device consists of a silicone rubber suction cup that is applied to the patient's chest and a cylinder mounted on two legs connected to a stiff back palate. It delivers compressions at a rate of 102 per minute and at a depth between 5 and $6 \mathrm{~cm}$ as prescribed in the guidelines for resuscitation provided by The European Resuscitation Council (ERC).

\section{Statistics}

Data management and statistical calculations were performed using STATA/SE 12.1. Normally distributed continuous variables are presented as mean \pm standard deviation and non-normally distributed variables as median and quartiles. Differences were analysed with Student's paired t-test or Wilcoxon's sign rank test as appropriate. Categorical variables are presented as number (n) and percent (\%) as appropriate, and differences are analysed with McNemar's test. The a priori $\alpha$-level was 0.05 .

\section{Results}

Data were reported on 696 OHCA patients who were attempted resuscitated between 1 October 2011 and 31 January 2013. Of these, 196 were resuscitated with both manual CPR and LUCAS CPR. Due to incomplete TTI data, 31 (4\%) patients were excluded. Another 41 (21\%) patients were excluded due to the fact that only data with LUCAS CPR were available. The remaining 155 OHCA patients comprised the study population (Figure 2).

Table 1 shows the baseline characteristics of the study population. The mean age was 66 years $(\mathrm{SD}=15)$. OHCA occurred particularly in men $(67 \%)$ at home $(81 \%)$ who presented a non-shockable rhythm at first rhythm analysis (64\%). OHCA was witnessed by either laypersons, health care professionals or EMS in 67\% (83/124) of the cases. Bystander CPR was provided in 74\% (93/125) of the cases. None of the patients in the study population were treated with a private or public AED before arrival of the EMS. The median time (interquartile range, IQR) from emergency call to EMS arrival was 5 (3-9) minutes, and the median time (IQR) from emergency call to first rhythm analysis was 9 (7-14) minutes.

Among the patients with a shockable rhythm as the presenting heart rhythm, the time (IQR) from emergency call to first defibrillation by the EMS was 9 (8-19) minutes.

CPR variables are shown in Tables 2 and 3. The mean NFF was significantly lower during the LUCAS episode $((16 \%$; $95 \%$ CI: $15 ; 18)$ than during the manual CPR episode $(35 \%$; $95 \%$ CI: $33 ; 37)(\mathrm{p}<0.001))$. In addition, the chest compression rate and the actual number of compressions per minute were significantly lower throughout the LUCAS episode than throughout the manual CPR episode.

There was no significant difference between the results of either NFT during rhythm analysis with and without defibrillation or pre- and post-shock NFT with manual 
Table 1 Baseline characteristics, $\mathbf{n}=155$

\begin{tabular}{|c|c|}
\hline Cardiac arrest background variables & \\
\hline Age in years, mean (SD) & $66( \pm 15), n=146$ \\
\hline Male gender $67 \%$ & $(92 / 138)$ \\
\hline Place of cardiac arrest & $81 \%(99 / 123)$ \\
\hline Home & $19 \%(23 / 123)$ \\
\hline Public & $1 \%(1 / 123)$ \\
\hline EMS vehicle & $81 \%(99 / 123)$ \\
\hline Cardiac arrest witnessed & $67 \%(83 / 124)$ \\
\hline Layperson & $53 \%(66 / 124)$ \\
\hline Health care professional & $5 \%(6 / 124)$ \\
\hline EMS & $9 \%(11 / 124)$ \\
\hline Bystander CPR & $74 \%(93 / 125)$ \\
\hline \multicolumn{2}{|l|}{ Rhythm on arrival of EMS } \\
\hline Asystole & $40 \%(50 / 126)$ \\
\hline PEA & $24 \%(31 / 126)$ \\
\hline VFNT & $33 \%(41 / 126)$ \\
\hline Other & $3 \%(4 / 126)$ \\
\hline Proportion of AED analysis & $98 \%(152 / 155)$ \\
\hline + defibrillation & $47 \%(73 / 155)$ \\
\hline - defibrillation & $87 \%(135 / 155)$ \\
\hline Defibrillation with AED before arrival of the EMS & $0 \%(0 / 155)$ \\
\hline
\end{tabular}

Continuous data presented as mean $+/-\mathrm{SD}$, valid cases.

Categorical variables presented as percentage ( $n / v a l i d$ cases).

$\mathrm{EMS}=$ Emergency medical service. Health care professional = EMS personnel, nurse, physician. $\mathrm{CPR}=$ cardiopulmonary resuscitation. $\mathrm{PEA}=$ Pulseless electrical activity. $\mathrm{VF}=$ Ventricular fibrillation. $\mathrm{VT}=$ Ventricular tachycardia. $\mathrm{AED}=$ Automated external defibrillator.

or LUCAS CPR (Table 3). LUCAS NFT was median $(\mathrm{IQR}=24(14-38))$ seconds.

Table 4 presents survival rates and treatment of hospitalised OHCA patients. Forty-five patients (29\%) were admitted to hospital alive and 14 (9\%) were discharged alive. Acute CAG was performed in $31(60 \%)$ of the patients. In six cases, the angiography was performed while LUCAS CPR was still being performed. Eight patients (15\%) underwent primary $\mathrm{PCI}$, and therapeutic hypothermia was induced in 27 (52\%) patients. Two (4\%) patients were treated with cardiopulmonary support. Both were admitted to hospital with on-going LUCAS CPR and both were alive after 30 days. In the remaining 103 (66\%) patients in whom CPR was deemed futile, treatment was terminated on the scene or upon admission to hospital. We found no differences in age or comorbidity among survivors and non-survivors.

\section{Discussion}

While recent years have seen studies reporting on the outcome associated with mechanical CPR $[7,14]$, only few studies have provided data on the quality of mechanical chest compressions, and those that have are limited by a low number of patients [15]. The present study evaluates the performance and quality of both manual and mechanical CPR in the same patient primarily based on TTI.

Our results show that during the manual episode, NFF lasted $34 \%$ of the time in patients without ROSC. However, once the LUCAS device was deployed, the NFF was reduced significantly to $16 \%$ of the time. Although an NFF of $34 \%$ during manual CPR is lower than what has been reported in previous studies where CPR was performed according to the former resuscitation guidelines of 2005 [4], we still find that the NFF is too high. Its size stresses the importance of short pauses during resuscitation and the importance of offering CPR training with performance feedback to further improve CPR quality. The average NFF of only $16 \%$ achieved with the LUCAS device was low. One could speculate that rhythm analysis was not performed every second minute as prescribed by the current guidelines. However, we have no data to substantiate this because the CODE-STAT does not allow registrations of the manual rhythm analysis. Thus, ambulance personnel have to use the automatic defibrillator mode as documented in the data derived from the defibrillator; inversely, physicians normally interpret the rhythm themselves to reduce CPR interruptions.

The low NFF with the LUCAS device may also have been achieved owing to fewer interruptions while loading the patient into the ambulance and during transport with on-going LUCAS.

Another advantage of the LUCAS device is that it affords the possibility of delivering shocks during compression and enables users to shorten or eliminate pauses for

Table 2 Quality of cardiopulmonary resuscitation (CPR)

\begin{tabular}{|c|c|c|c|}
\hline \multirow[t]{2}{*}{ CPR variables } & \multirow{2}{*}{$\begin{array}{l}\text { Manual CPR } \\
\mathrm{n}=155\end{array}$} & \multirow{2}{*}{$\begin{array}{l}\text { LUCAS CPR } \\
n=155\end{array}$} & \multirow[t]{2}{*}{ P-value } \\
\hline & & & \\
\hline Episode duration, min. & $5(2 ; 6)$ & $13(11 ; 14)$ & $<0.001$ \\
\hline No-flow fraction, $\%$ & $35(33 ; 37)$ & $16(15 ; 18)$ & $<0.001$ \\
\hline Chest compression rate per minute & $124(121 ; 126)$ & $102(102 ; 102)$ & $<0.001$ \\
\hline Number of chest compressions per minute & $75(72 ; 79)$ & $84(82 ; 85)$ & $<0.001$ \\
\hline
\end{tabular}

No-flow time $=$ Time without ROSC - time without chest compressions.

No-flow fraction $=$ No-flow time/(episode duration - time with ROSC). ROSC $=$ Return of spontaneous circulation.

CPR variables are presented as mean values $(95 \% \mathrm{Cl})$. 
Table 3 No-flow time (NFT) and rhythm analysis with/without defibrillation

\begin{tabular}{|c|c|c|c|c|c|}
\hline \multirow[t]{2}{*}{ CPR variables } & \multicolumn{2}{|l|}{ Manual CPR } & \multicolumn{3}{|l|}{ LUCAS CPR } \\
\hline & Mean $(95 \% \mathrm{Cl})$ sec. & Number with data & Mean $(95 \% \mathrm{Cl})$ sec. & Number with data & P-value \\
\hline NFT in relation to AED analysis & $15(13 ; 18)$ & 60 & $16(13 ; 19)$ & 55 & 0.620 \\
\hline \multicolumn{6}{|l|}{ + defibrillation } \\
\hline NFT in relation to AED analysis & $17(16 ; 18)$ & 112 & $18(16 ; 20)$ & 101 & 0.960 \\
\hline \multicolumn{6}{|l|}{ - defibrillation } \\
\hline Pre-shock NFT & $17(15 ; 20)$ & 60 & $20(16 ; 23)$ & 55 & 0.406 \\
\hline Post-shock NFT & $7(6 ; 8)$ & 60 & $7(6 ; 9)$ & 55 & 0.466 \\
\hline
\end{tabular}

No-flow time $(\mathrm{NFT})=$ Time without ROSC - time without chest compressions. ROSC $=$ Return of spontaneous circulation. AED $=$ Automated external defibrillator.

defibrillation. Nevertheless, we found no difference in preshock and post-shock NFT in relation to automated rhythm analysis throughout the time with manual CPR or LUCAS CPR. The median pre-shock pause was $17 \mathrm{sec}$. (IQR 15-20) and the median post-shock pause was $7 \mathrm{sec}$. (IQR 6-8). While these pauses are reasonable for defibrillation in automatic mode during manual CPR and similar to pauses reported in previous research [12], they are suboptimal for defibrillation during LUCAS CPR. However, the TTI analysis of all cases at the level of single chest compression revealed that the pre-shock and the postshock NFT during LUCAS CPR might be overestimated due to the inability of CODE-STAT to register chest compressions when shock is delivered. The chest compression rate and the actual number of compressions per minute, which are important elements in CPR quality, were significantly better performed during LUCAS CPR. The chest compressions were delivered at a rate higher than recommended, and they were probably also too shallow during manual CPR (124 compressions per minute), which may result in failure to achieve ROSC.

Although they fell short of being optimal, the manual chest compression rate and the number of chest compressions per minute still appear superior to the manual compression rates (138 compressions per minute) reported in the study by Krarup et al. [4].

The episode duration with LUCAS CPR was nearly three times longer than the manual CPR episode (Table 3). This agrees well with previous reports, and it presumably reflects that these cases are the ones with the longest resuscitation attempt [16].
In a study of changes in bystander resuscitation attempts and survival during a 10 -year period in which national initiatives were taken to improve rates of bystander CPR, Wissenberg et al. [17] concluded that an increase in survival following OHCA was associated with an increase in bystander CPR, regardless of witnessed cardiac arrest. This is consistent with the findings in our study, which showed a large proportion of patients with cardiac arrest at home and a high rate of bystander CPR. Our study was not designed or statistically powered to evaluate survival outcome. Nevertheless, the 30-day survival after OHCA resembles the previously described values of $9 \%[17,18]$. This was achieved despite the fact that the present cohort may consist of a selected high-risk group in need of prolonged CPR as compared with patients who are easily resuscitated within a few minutes after start of CPR or defibrillation. The two patients admitted to hospital with on-going LUCAS CPR and who were treated with cardiopulmonary support were resuscitated during a particularly long time; regardless of this, both patients were alive after 30 days with minimal neurological sequelae. We believe that it is unlikely that these patients would have survived if transported and treated with manual CPR only. Furthermore, manual CPR is cumbersome during catheterisation and potentially hazardous for the health care personnel due to radiation exposure. Therefore, the LUCAS device seems ideal during catheterisation compared with manual CPR [19].

\section{Limitations}

There are some limitations to this study. First, it is a small observational, prospective study with potentially

Table 4 In-hospital treatment among patients admitted alive $(n=45)$ and among patients admitted with on-going LUCAS CPR $(n=7), n=52$

\begin{tabular}{llll}
\hline Treatment & Admitted alive $(\mathbf{n}=\mathbf{4 5})$ & Ongoing LUCAS CPR $(\mathbf{n}=\mathbf{7})$ & Total $(\mathbf{n}=\mathbf{5 2})$ \\
\hline Coronary angiography & $56 \%(25)$ & $86 \%(6)$ & $60 \%(31)$ \\
Percutaneous coronary intervention & $16 \%(7)$ & $14 \%(1)$ & $15 \%(8)$ \\
Therapeutic hypothermia & $58 \%(26)$ & $14 \%(1)$ & $52 \%(27)$ \\
Cardiopulmonary support & $0 \%(0)$ & $29 \%(2)$ & $4 \%(2)$ \\
\hline
\end{tabular}

Categorical variables presented as percentage (valid cases). 
unknown confounders; however, the relative advantage of this study design is that being their own controls, the patients studied are the same during manual CPR and LUCAS CPR. Second, the physician of the prehospital critical care team decides if the LUCAS device is to be used in the resuscitation, which undoubtedly introduces selection bias. Third, even though TTI and ECG data transfer to the central server was mandatory, a large part of the OHCA data was never transferred to the server.

Ventilation and compression depth measurements during resuscitation are recommended for the evaluation of CPR quality; however, these measurements cannot be assessed from TTI data alone, and little data support the clinical importance of these variables in this setting [13]. The prehospital critical care teams were to take part in filling out a separate study form regarding the end-tidal $\mathrm{CO}_{2}\left(\mathrm{ETCO}_{2}\right)$, which is considered a surrogate measure of cardiac output during CPR [20]; however, little evidence exists to support this concept. Unfortunately, we are not able to report the $\mathrm{ETCO}_{2}$ values in this study due to the lack of registered values/too many missing values. Data on adverse device events or injuries have likewise not been reported in those patients not surviving OHCA due to the fact that these events were not described in the medical reports. The 45 patients admitted to hospital alive did not have any device-related events or injuries.

\section{Conclusion}

Mechanical chest compressions provided by the LUCAS device improve CPR quality by significantly reducing the NFF and by improving the quality of chest compression compared with manual CPR during OHCA resuscitation.

\section{Perspectives}

Randomised clinical studies not have been able to demonstrate improved survival for patients resuscitated with mechanical chest compression devices, and the devices have to some extent been discredited following the ASPIRE trial, the LINC trial and the PARAMEDIC trial. However, these trials are impeded by methodological deficiencies such as different resuscitation protocols in the compared groups and, in particular, prolonged time to deployment of the device. Maintenance of high-quality CPR during OHCA is not easy because of the small number of crew present, fatigue, patient access, and the impossibility of performing resuscitation in a moving vehicle. These considerations must be balanced against the current evidence from previous studies when deciding on the future role of mechanical CPR in healthcare systems, but it seems reasonably that such devices will continue to play a role when manual CPR is impractical. Nevertheless, it is essential that resources are available to support regular training of healthcare personnel ensuring that resuscitation guidelines are followed and to monitor CPR quality continuously.

\section{Competing interests}

The authors declare that they have no competing interests.

\section{Authors' contributions}

TT carried out collection of data, data management, interpretation of the data/ results and drafted the manuscript. JFL participated in the design of the study and critically revised the manuscript. AKK critically revised the manuscript. $\mathrm{TMH}$ participated in the collection of data and revision of the manuscript. CS participated in the collection of data. LK participated in the collection of data. ST participated in the collection of data. CJT conceived and designed the study, contributed to the interpretation of the results, critically revised the manuscript and helped to draft the manuscript. All authors have read and approved the final manuscript.

\section{Authors' information}

TT; MD, PhD research fellow, Aarhus University Hospital, Denmark. JFL; MD, PhD Department of Cardiology, Aarhus University Hospital, Denmark. TMH; MD, head of Prehospital Medical Care Service and Helicopter Emergency Medical Service Central Denmark Region. CS; MD, PhD Department of Cardiology Aarhus University Hospital, Denmark. LK; MD, Helicopter Emergency Medical Service Central Denmark Region. ST; MD, Quality head of pre-hospital medical care, Falck A/S, Denmark. CJT; MD, PhD, DSc Department of Cardiology, Aarhus University Hospital, Denmark.

\section{Acknowledgements}

The authors would like to thank all paramedics, nurses and physicians of the EMS, including the PMA and the HEMS for their collaboration in transferring TTI data. Physio-Control Inc. is thanked for providing the software necessary to analyse data and for providing equipment for the study.

\section{Founding sources}

The Danish Heart Foundation, The Riisfort Foundation, The Simon Fougner Hartmann Foundation, The Arvid Nielson Foundation and The Savvaerksejer Foundation supported the study.

\section{Author details}

${ }^{1}$ Department of Cardiology, Aarhus University Hospital, Aarhus, Denmark. ${ }^{2}$ Department of Prehospital Medical Care Service, Central Denmark Region, Aarhus, Denmark. ${ }^{3}$ Prehospital Critical Care Team, Aarhus University Hospital, Aarhus, Denmark. ${ }^{4}$ Helicopter Emergency Medical Service, Central Denmark Region, Aarhus, Denmark. ${ }^{5}$ Falck A/S, Copenhagen, Denmark.

Received: 12 January 2015 Accepted: 9 April 2015

Published online: 22 April 2015

\section{References}

1. Edelson DP, Abella BS, Kramer-Johansen J, Wik L, Myklebust H, Barry AM, et al. Effects of compression depth and pre-shock pauses predict defibrillation failure during cardiac arrest. Resuscitation. 2006;71:137-45.

2. Deakin CD, Nolan JP, Soar J, Sunde K, Koster RW, Smith GB, et al. European Resuscitation Council Guidelines for Resuscitation 2010 Section 4. Adult advanced life support. Resuscitation. 2010;81:1305-52.

3. Wik L, Kramer-Johansen J, Myklebust H, Sorebo H, Svensson L, Fellows B, et al. Quality of cardiopulmonary resuscitation during out-of-hospital cardiac arrest. JAMA. 2005;293:299-304.

4. Krarup NH, Terkelsen CJ, Johnsen SP, Clemmensen P, Olivecrona GK, Hansen TM, et al. Quality of cardiopulmonary resuscitation in out-of-hospital cardiac arrest is hampered by interruptions in chest compressions-A nationwide prospective feasibility study. Resuscitation. 2011;82:263-9.

5. Bonnemeier $H$, Simonis $G$, Olivecrona $G$, Weidtmann B, Gotberg M, Weitz G, et al. Continuous mechanical chest compression during in-hospital cardiopulmonary resuscitation of patients with pulseless electrical activity. Resuscitation. 2011;82:155-9.

6. Wagner H, Madsen Hardig B, Steen S, Sjoberg T, Harnek J, Olivecrona GK. Evaluation of coronary blood flow velocity during cardiac arrest with circulation maintained through mechanical chest compressions in a porcine model. BMC Cardiovasc Disord. 2011;11:73. 
7. Rubertsson S, Lindgren E, Smekal D, Ostlund O, Silfverstolpe J, Lichtveld RA, et al. Mechanical chest compressions and simultaneous defibrillation vs conventional cardiopulmonary resuscitation in out-of-hospital cardiac arrest: the LINC randomized trial. JAMA. 2014;311:53-61.

8. Wik L, Olsen J, Persse D, Sterz F, Lozano Jr M, Brouwer MA, et al. Manual vs. integrated automatic load-distributing band CPR with equal survival after out of hospital cardiac arrest. The randomized CIRC trial. Resuscitation. 2014:85:741-8

9. Axelsson C, Nestin J, Svensson L, Axelsson AB, Herlitz J. Clinical consequences of the introduction of mechanical chest compression in the EMS system for treatment of out-of-hospital cardiac arrest-a pilot study. Resuscitation. 2006;71:47-55.

10. Jacobs I, Nadkarni V, Bahr J, Berg RA, Billi JE, Bossaert L, et al. Cardiac arrest and cardiopulmonary resuscitation outcome reports: update and simplification of the Utstein templates for resuscitation registries. A statement for healthcare professionals from a task force of the international liaison committee on resuscitation (American Heart Association, European Resuscitation Council, Australian Resuscitation Council, New Zealand Resuscitation Council, Heart and Stroke Foundation of Canada, InterAmerican Heart Foundation, Resuscitation Council of Southern Africa). Resuscitation. 2004;63:233-49.

11. Stecher FS, Olsen JA, Stickney RE, Wik L. Transthoracic impedance used to evaluate performance of cardiopulmonary resuscitation during out of hospital cardiac arrest. Resuscitation. 2008;79:432-7.

12. Yost D, Phillips RH, Gonzales L, Lick CJ, Satterlee P, Levy M, et al. Assessment of CPR interruptions from transthoracic impedance during use of the LUCAS ${ }^{\text {TM }}$ mechanical chest compression system. Resuscitation. 2012;83:961-5.

13. Kramer-Johansen J, Edelson DP, Losert H, Kohler K, Abella BS. Uniform reporting of measured quality of cardiopulmonary resuscitation (CPR). Resuscitation. 2007;74:406-17.

14. Hallstrom A, Rea TD, Sayre MR, Christenson J, Anton AR, Mosesso Jr VN, et al. Manual chest compression vs use of an automated chest compression device during resuscitation following out-of-hospital cardiac arrest: a randomized trial. JAMA. 2006;295:2620-8.

15. Putzer G, Braun P, Zimmermann A, Pedross F, Strapazzon G, Brugger $H$, et al. LUCAS compared to manual cardiopulmonary resuscitation is more effective during helicopter rescue-a prospective, randomized, cross-over manikin study. Am J Emerg Med. 2013;31:384-9.

16. Olasveengen TM, Wik L, Steen PA. Quality of cardiopulmonary resuscitation before and during transport in out-of-hospital cardiac arrest. Resuscitation. 2008;76:185-90.

17. Wissenberg M, Lippert FK, Folke F, Weeke P, Hansen CM, Christensen EF, et al. Association of national initiatives to improve cardiac arrest management with rates of bystander intervention and patient survival after out-of-hospital cardiac arrest. JAMA. 2013;310:1377-84.

18. Nichol G, Thomas E, Callaway CW, Hedges J, Powell JL, Aufderheide TP, et al. Regional variation in out-of-hospital cardiac arrest incidence and outcome. JAMA. 2008;300:1423-31.

19. Wagner H, Terkelsen C, Friberg H, Harnek J, Kern K, Lassen JF, et al. Cardiac arrest in the catheterisation laboratory: a 5-year experience of using mechanical chest compressions to facilitate PCI during prolonged resuscitation efforts. Resuscitation. 2010;81:383-7.

20. Sheak KR, Wiebe DJ, Leary M, Babaeizadeh S, Yuen TC, Zive D, et al. Quantitative relationship between end-tidal carbon dioxide and CPR quality during both in-hospital and out-of-hospital cardiac arrest. Resuscitation. 2015;89C:149-54.

\section{Submit your next manuscript to BioMed Central and take full advantage of:}

- Convenient online submission

- Thorough peer review

- No space constraints or color figure charges

- Immediate publication on acceptance

- Inclusion in PubMed, CAS, Scopus and Google Scholar

- Research which is freely available for redistribution 